\title{
CARACTERIZAÇÃO DO MEL PRODUZIDO POR ESPÉCIES DE Melipona Illiger, 1806 (APIDAE: MELIPONINI) DA REGIÃO NORDESTE DO BRASIL: 1. CARACTERÍSTICAS FÍSICO-QUÍMICAS
}

\author{
Bruno de Almeida Souza* \\ Núcleo de Pesquisas com Abelhas, Embrapa Meio-Norte, CP 01, 64006-220 Teresina - PI, Brasil \\ Luís Carlos Marchini \\ Departamento de Entomologia, Fitopatologia e Zoologia Agrícola, Escola Superior de Agricultura "Luiz de Queiroz", \\ Universidade de São Paulo, CP 09, 13418-900 Piracicaba - SP, Brasil \\ Melissa Oda-Souza \\ Departamento de Ciências Florestais, Escola Superior de Agricultura "Luiz de Queiroz", Universidade de São Paulo, \\ CP 09, 13418-900 Piracicaba - SP, Brasil
}

Carlos Alfredo Lopes de Carvalho

Centro de Ciências Agrárias, Ambientais e Biológicas, Universidade Federal do Recôncavo da Bahia, CP 118, 44380-000 Cruz das Almas - BA, Brasil

Rogério Marcos de Oliveira Alves

Escola Agrotécnica Federal de Catu, Rua Barão de Camaçari, 118, 48110-000 Catu - BA, Brasil

Recebido em 16/1/08; aceito em 8/9/08; publicado na web em 5/2/09

\begin{abstract}
CHARACTERIZATION OF HONEY PRODUCED BY SPECIES OF Melipona Illiger, 1806 (APIDAE: MELIPONINI) FROM THE NORTHEAST AREA OF BRAZIL: 1. PHYSICO-CHEMICAL CHARACTERISTICS. Thirty three honey samples produced by four Melipona species from different areas of the State of Bahia, were analyzed with the aim to determine their physico-chemical characteristics, contributing to the establishment of standards for quality control. The majority of the average values for physicochemical parameters fulfilled the quality criteria established by the Brazilian and international Legislations for Apis honey, except for moisture content, which afforded higher values. Concerning the high number of samples wich did not fit the limits for reducing sugars, it is necessary to define minimum values in order to characterize Melipona honeys, as well as criteria for use of diastasic activity.
\end{abstract}

Keywords: stingless bee; physico-chemical characteristics; Melipona sp.

\section{INTRODUÇÃO}

O mel dos meliponíneos é um produto que tem apresentado uma demanda crescente de mercado, obtendo preços mais elevados que o das abelhas do gênero Apis. Mesmo assim, ainda são poucos os estudos sobre as suas características físico-químicas, que auxiliem na definição de padrões de qualidade para sua comercialização, sendo que a maioria dos trabalhos que objetivam ao maior conhecimento deste produto leva em consideração padrões e características estabelecidas para o mel de A. mellifera.

No entanto, a tentativa de aplicar estas normatizações internacionais, ou mesmo nacionais, estabelecidas para o mel de A. mellifera tem a possibilidade de gerar problemas quanto à avaliação da qualidade do mel produzido pelos meliponíneos. Além disto, Fallico et al. ${ }^{1}$ citam modificações feitas pela Codex Alimentarius Commission (CAC) na definição do produto mel, sendo considerado como a "substância natural, doce produzida por todas as abelhas melíferas a partir de néctar de plantas ou de secreções de partes vivas de plantas...", incluindo-se nesta definição as demais espécies de abelhas, e não somente $A$. mellifera.

A partir da abrangência proporcionada por esta nova definição para o produto, a International Honey Commission (IHC) criou um grupo de trabalho para a definição de padrões de qualidade para produtos das abelhas, onde se incluiu o mel produzido pelas abelhas sem ferrão. Neste sentido, diversos estudos vêm sendo realizados no sentido de construir uma base de dados para auxiliar nestas definições. ${ }^{2-6}$

*e-mail: bruno@cpamn.embrapa.br
Desta forma, o objetivo do presente trabalho foi determinar as características físico-químicas de amostras de mel produzido por espécies de Melipona na região nordeste do Brasil, contribuindo com informações para o estabelecimento de normas para seu controle de qualidade.

\section{PARTE EXPERIMENTAL}

Foram utilizadas 33 amostras de mel produzido pelas seguintes espécies do gênero Melipona: Melipona asilvai (7 amostras), $M$. mandacaia (2 amostras), M. quadrifasciata anthidioides ( 9 amostras) e M. scutellaris (15 amostras) (Tabela 1). As amostras foram colhidas entre o período de dezembro de 2004 e maio de 2006, provenientes de diferentes localidades do Estado da Bahia, região nordeste do Brasil, em suas respectivas épocas de produção.

As amostras foram obtidas por meio de sucção com seringa descartável ou bomba elétrica, sendo armazenadas em potes plásticos com fechamento hermético e mantidas sob refrigeração (aproximadamente $5,0{ }^{\circ} \mathrm{C}$ ) até a realização das análises no Laboratório de Insetos Úteis, setor de Entomologia do Departamento de Entomologia, Fitopatologia e Zoologia Agrícola da Escola Superior de Agricultura "Luiz de Queiroz" - USP, campus de Piracicaba, Estado de São Paulo, Brasil.

Foram realizadas as seguintes análises físico-químicas: umidade, atividade diastásica, $\mathrm{pH}$ e acidez livre, índice de formol, cinzas, cor, viscosidade, condutividade elétrica, açúcares redutores, açúcares redutores totais e sacarose, hidroximetilfurfural, proteína e atividade de água $\left(a_{w}\right){ }^{7,8}$ 
Tabela 1. Locais de colheita (localidade e coordenadas), espécies de abelhas produtoras e codificação das amostras de mel de Melipona (Apidae: Meliponini) do Estado da Bahia, região nordeste do Brasil

\begin{tabular}{lccc}
\hline Locais & Coordenadas & Espécies & Amostras \\
\hline Camaçari & $12^{\circ} 58^{\prime} \mathrm{S} / 38^{\circ} 15^{\prime} \mathrm{O}$ & M. scutellaris & $7,14,16$ \\
Cruz das Almas & $12^{\circ} 40^{\prime} \mathrm{S} / 39^{\circ} 06^{\prime} \mathrm{O}$ & M. scutellaris & 17,20 \\
& & M. quadrifasciata anthidioides & $28,30,31,33$ \\
Itaberaba & $12^{\circ} 31^{\prime} \mathrm{S} / 40^{\circ} 18^{\prime} \mathrm{O}$ & M. asilvai & 32 \\
& & M. quadrifasciata anthidioides & $4,5,6,8,9,10,11,12,13,15,18$ \\
Porto de Sauípe & $12^{\circ} 22^{\prime} \mathrm{S} / 37^{\circ} 53^{\prime} \mathrm{O}$ & M. scutellaris & 29 \\
Quijingue & $10^{\circ} 45^{\prime} \mathrm{S} / 39^{\circ} 13^{\prime} \mathrm{O}$ & M. quadrifasciata anthidioides & $21,22,23$ \\
Salgadália & $11^{\circ} 27^{\prime} \mathrm{S} / 39^{\circ} 10^{\prime} \mathrm{O}$ & M. asilvai & $24,25,26,27$ \\
São Gabriel & & M. quadrifasciata anthidioides & 1,2 \\
Serrinha & $11^{\circ} 13^{\prime} \mathrm{S} / 41^{\circ} 54^{\prime} \mathrm{O}$ & M. mandacaia & 3
\end{tabular}

Os valores médios obtidos de três repetições foram comparados às normas nacionais e internacionais existentes para mel de A. mellifera, e também com a norma proposta para mel de Meliponinae do Brasil. Especificamente para atividade diastásica, em nenhuma das amostras de mel de Melipona analisadas foi conseguida leitura para a atividade desta enzima, sendo este resultado discutido no item específico.

\section{RESULTADOS E DISCUSSÃO}

Os resultados obtidos para as análises físico-químicas das 33 amostras de mel de abelhas sem ferrão são apresentados na Tabela 2. Valores de acidez livre, HMF, açúcares (redutores totais, redutores e sacarose) e umidade são apresentados com só um decimal, como sugerido por Bogdanov et al.. ${ }^{9}$

Considerando a revisão sobre as características físico-químicas do mel de abelhas sem ferrão, recentemente publicada por Souza et al., ${ }^{2}$ as informações obtidas no presente trabalho serão discutidas principalmente com base nesta referência e em outros trabalhos publicados após este artigo, mesmo os relacionados a outros gêneros de meliponíneos. Ressalta-se que apesar do grande impulso que vem sendo dado ao conhecimento deste produto das abelhas sem ferrão, muitas das informações existentes estão publicadas em resumos de anais de eventos e reuniões científicas, com poucas publicações em periódicos científicos.

\section{Umidade}

Os valores de umidade variaram de 26,8 a $43,8 \%$ nas amostras de mel de Melipona, sendo constatada uma reprovação de todas as amostras por apresentarem esta característica acima do máximo de $20 \%$ permitido pelas legislações brasileira ${ }^{10} \mathrm{e}$ internacional ${ }^{11}$ para mel. Estes elevados valores para umidade podem ser considerados como regra para mel de meliponíneos, influenciando outras características como viscosidade, fluidez e conservação.

Esta umidade elevada já foi constatada para M. asilvai, ${ }^{12} M$. compressipes,${ }^{6,13} M$. favosa,${ }^{14} M$. mandacaia,${ }^{15} M$. quadrifasciata, ${ }^{16}$ M. scutellaris, ${ }^{17,18}$ M. seminigra ${ }^{19}$ e Tetragonisca angustula ${ }^{20,21}$ Os autores determinaram valores de umidade variando de 16,7 a 45,0\%, considerados extremamente elevados quando comparados com a variação normalmente apresentada por mel de Apis.

\section{Atividade diastásica}

Não foi conseguida leitura para a atividade diastásica nas amotras de mel de Melipona. Atualmente o valor mínimo aceito pelas normas internacional e brasileira é de 3,0 Gothe. A principal relevância desta enzima diz respeito à sua sensibilidade ao calor, sendo recomendada para avaliar a qualidade do mel, fornecendo indicações sobre o grau de conservação e superaquecimento do produto. ${ }^{22}$

No geral, as informações existentes sobre esta enzima a consideram como de baixa atividade em espécies de Melipona. Vit e Pulcini2 ${ }^{23}$ encontraram 2,6 Gothe como o menor valor em amostras de mel das espécies $M$. compressipes compressipes, $M$. favosa favosa, M. lateralis kangarumensis, M. paraensis e Scaptotrigona. O maior valor (35,6 Gothe) foi determinado por estes autores em amostras de mel da tribo Trigonini. Valores compilados por Souza et $a .^{2}{ }^{2}$ variaram entre 0,9 e 23,0 Gothe para diversas espécies de meliponíneos. Já Almeida-Anacleto ${ }^{21}$ encontrou variações de 3,16 a 54,11 Gothe para espécies de trigoníneos de Piracicaba. Para méis de T. angustula, Almeida-Muradian e Barion ${ }^{5}$ encontraram atividade diastásica de 16,6 Gothe.

Esta inconstância dos resultados obtidos para atividade diastásica, também verificada para mel de Apis, levou White Júnior ${ }^{24}$ a questionar o uso desta enzima como um indicador de qualidade do mel, devido à grande variação na quantidade desta enzima em méis recém-colhidos e não aquecidos, sugerindo a exclusão desta análise por ser um teste redundante, enganoso e variável.

Mesmo na presença destas opiniões divergentes, há o consenso sobre a baixa atividade desta enzima em mel de Melipona assim, ao invés de objetivar a verificação da qualidade do mel produzido, a diastase poderia vir a se constituir em um marcador para mel produzido por este gênero de abelhas, atestando a sua origem entomológica. Para isso são necessários ainda mais estudos sobre a produção desta enzima pelas abelhas sem ferrão.

\section{pH, acidez livre e índice de formol}

Os valores de $\mathrm{pH}$ e acidez livre determinados variaram entre 3,16 e 6,50, e 5,1 e 88,6 meq kg-1, respectivamente. Destaca-se o valor de $\mathrm{pH}$ determinado para a amostra 7, de 6,50, chegando quase a atingir a neutralidade. No total, 10 amostras, correspondendo a 30,3\%, foram consideradas impróprias para consumo humano direto, com base no valor máximo de 50,0 meq $\mathrm{kg}^{-1}$ para acidez livre estabelecido pelas normas brasileira e internacional para mel. Para pH não há valores de referência estabelecidos.

$\mathrm{O}$ pH e a acidez são considerados importantes fatores antimicrobianos, provendo maior estabilidade ao produto quanto ao 
Tabela 2. Características físico-químicas de amostras de mel de meliponíneos (Apidae: Meliponini) provenientes da região nordeste do Brasil

\begin{tabular}{|c|c|c|c|c|c|c|c|c|c|c|c|c|c|c|c|}
\hline Espécies & Amo & Umi & $\mathrm{pH}$ & Aci & IF & Cin & Cor & Vis & Cond & AT & $\mathrm{AR}$ & $\mathrm{Sac}$ & HMF & Pro & $a_{w}$ \\
\hline \multirow{7}{*}{ M. asilvai } & 28 & 41,2 & 3,25 & 74,7 & 6,05 & 0,05 & 0,300 & 20,0 & 362,0 & 59,3 & 57,2 & 2,1 & 3,8 & 0,50 & 0,824 \\
\hline & 30 & 39,8 & 3,46 & 42,0 & 9,01 & 0,06 & 0,236 & 10,0 & 351,0 & 63,2 & 59,3 & 3,5 & 42,8 & 0,28 & 0,797 \\
\hline & 31 & 41,4 & 3,23 & 72,4 & 9,05 & 0,05 & 0,298 & 30,0 & 410,7 & 61,0 & 59,5 & 1,4 & 4,6 & 0,08 & 0,812 \\
\hline & 33 & 43,8 & 3,16 & 70,4 & 6,03 & 0,01 & 0,272 & 10,0 & 374,7 & 57,9 & 55,8 & 1,9 & 9,0 & 0,78 & 0,851 \\
\hline & 21 & 31,5 & 4,08 & 25,3 & 7,08 & 0,13 & 0,163 & 20,0 & 665,5 & 59,7 & 56,3 & 3,2 & 0,8 & 0,21 & 0,759 \\
\hline & 22 & 34,0 & 3,72 & 63,3 & 7,03 & 0,18 & 0,216 & 50,0 & 871,5 & 60,1 & 50,6 & 9,0 & 7,7 & 0,23 & 0,746 \\
\hline & 23 & 31,0 & 3,96 & 31,5 & 7,11 & 0,16 & 0,233 & 40,0 & 790,0 & 95,6 & 93,1 & 2,3 & 34,3 & 0,25 & 0,754 \\
\hline \multirow{2}{*}{ M. mandacaia } & 1 & 31,8 & 4,14 & 19,0 & 11,01 & 0,09 & 0,154 & 80,0 & 255,0 & 75,2 & 74,7 & 0,4 & 1,5 & 0,25 & 0,757 \\
\hline & 2 & 31,0 & 3,27 & 56,4 & 6,04 & 0,08 & 0,344 & 120,0 & 312,3 & 81,9 & 76,3 & 5,3 & 60,2 & 0,09 & 0,740 \\
\hline \multirow{9}{*}{$\begin{array}{l}\text { M. quadrifasciata } \\
\text { anthidioides }\end{array}$} & 32 & 35,8 & 3,22 & 88,6 & 5,03 & 0,09 & 0,167 & 60,0 & 506,3 & 64,7 & 62,8 & 1,8 & 6,0 & 0,36 & 0,777 \\
\hline & 3 & 33,0 & 4,62 & 24,1 & 5,02 & 0,17 & 0,171 & 20,0 & 591,3 & 81,8 & 76,8 & 4,8 & 22,6 & 0,30 & 0,776 \\
\hline & 29 & 31,0 & 3,46 & 56,0 & 9,01 & 0,07 & 0,602 & 70,0 & 620,0 & 68,4 & 66,5 & 1,8 & 18,8 & 0,08 & 0,738 \\
\hline & 19 & 29,5 & 3,53 & 62,3 & 7,04 & 0,13 & 0,669 & 100,0 & 682,0 & 64,1 & 56,2 & 7,4 & 32,1 & 0,49 & 0,725 \\
\hline & 24 & 31,0 & 4,23 & 20,1 & 4,02 & 0,12 & 0,396 & 50,0 & 527,0 & 83,4 & 80,9 & 2,4 & 23,2 & 0,11 & 0,765 \\
\hline & 20 & 31,0 & 3,48 & 64,3 & 5,02 & 0,08 & 0,577 & 40,0 & 535,5 & 83,6 & 83,2 & 0,3 & 4,7 & 0,22 & 0,749 \\
\hline & 25 & 32,5 & 4,72 & 14,3 & 3,06 & 0,06 & 0,402 & 10,0 & 389,5 & 67,1 & 60,2 & 6,6 & 12,2 & 0,19 & 0,784 \\
\hline & 26 & 33,0 & 4,28 & 18,2 & 4,04 & 0,12 & 0,373 & 50,0 & 504,5 & 65,4 & 64,7 & 0,7 & 21,7 & 0,60 & 0,770 \\
\hline & 27 & 32,0 & 4,34 & 17,2 & 4,04 & 0,09 & 0,363 & 30,0 & 583,5 & 65,4 & 63,3 & 2,0 & 3,1 & 0,23 & 0,763 \\
\hline \multirow{15}{*}{ M. scutellaris } & 4 & 30,3 & 5,42 & 8,1 & 6,05 & 0,29 & 0,172 & 90,0 & 728,7 & 78,3 & 77,1 & 1,1 & 1,5 & 0,34 & 0,722 \\
\hline & 5 & 29,1 & 4,08 & 15,2 & 7,06 & 0,20 & 0,156 & 130,0 & 572,3 & 71,4 & 71,1 & 0,2 & 0,8 & 0,30 & 0,712 \\
\hline & 6 & 29,4 & 4,68 & 12,1 & 8,01 & 0,24 & 0,151 & 120,0 & 685,0 & 71,9 & 71,6 & 0,4 & 0,8 & 0,08 & 0,715 \\
\hline & 7 & 28,8 & 6,50 & 5,1 & 5,05 & 0,21 & 0,128 & 130,0 & 485,7 & 89,2 & 87,2 & 1,9 & 8,4 & 1,21 & 0,711 \\
\hline & 8 & 28,8 & 4,56 & 17,2 & 16,12 & 0,20 & 0,180 & 160,0 & 615,0 & 68,0 & 67,8 & 0,2 & 0,0 & 0,04 & 0,690 \\
\hline & 9 & 27,4 & 4,32 & 16,1 & 7,05 & 0,18 & 0,249 & 220,0 & 586,3 & 62,3 & 60,5 & 1,7 & 0,0 & 0,04 & 0,662 \\
\hline & 10 & 28,7 & 4,58 & 13,0 & 7,00 & 0,22 & 0,111 & 140,0 & 588,7 & 66,2 & 59,7 & 6,2 & 0,8 & 0,09 & 0,681 \\
\hline & 11 & 30,2 & 4,02 & 18,2 & 6,06 & 0,11 & 0,122 & 110,0 & 500,7 & 69,6 & 69,0 & 0,5 & 0,0 & 0,04 & 0,686 \\
\hline & 12 & 27,7 & 4,42 & 16,2 & 6,06 & 0,24 & 0,235 & 220,0 & 564,7 & 73,0 & 72,8 & 0,2 & 0,8 & 0,25 & 0,669 \\
\hline & 13 & 31,0 & 4,24 & 18,0 & 5,00 & 0,18 & 0,158 & 80,0 & 534,0 & 59,7 & 57,8 & 1,8 & 0,0 & 0,46 & 0,709 \\
\hline & 14 & 31,0 & 3,90 & 53,3 & 9,06 & 0,33 & 0,356 & 50,0 & 905,7 & 59,5 & 56,7 & 2,7 & 12,9 & 0,30 & 0,736 \\
\hline & 15 & 28,0 & 3,98 & 18,2 & 7,07 & 0,15 & 0,193 & 150,0 & 540,0 & 75,0 & 70,0 & 4,7 & 0,0 & 0,09 & 0,708 \\
\hline & 16 & 29,8 & 3,83 & 34,2 & 7,04 & 0,08 & 0,540 & 150,0 & 355,7 & 95,2 & 92,5 & 2,6 & 0,8 & 0,17 & 0,720 \\
\hline & 17 & 26,8 & 4,24 & 24,0 & 8,01 & 0,14 & 0,324 & 120,0 & 556,3 & 84,3 & 83,5 & 0,8 & 2,3 & 0,18 & 0,734 \\
\hline & 18 & 30,0 & 3,62 & 29,1 & 5,01 & 0,06 & 0,122 & 10,0 & 334,3 & 65,6 & 63,3 & 2,2 & 0,8 & 0,17 & 0,743 \\
\hline
\end{tabular}

Amo: Amostra; Umi: Umidade (\%); Aci: Acidez livre (meq kg-1); IF: Índice de formol ( $\left.\mathrm{mL} \mathrm{kg}^{-1}\right)$; Cin: cinzas (\%); Vis: Viscosidade (mPa s); Cond: Condutividade elétrica $\left(\mu \mathrm{S} \mathrm{cm}{ }^{-1}\right)$; AT: Açúcares redutores totais (\%); AR: Açúcares redutores (\%); Sac: Sacarose (\%); HMF: Hidroximetilfurfural ( $\left.\mathrm{mg} \mathrm{kg}^{-1}\right)$; Pro: Proteína (\%); $a_{w}$ : atividade de água.

desenvolvimento de microrganismos. Mesmo com a presença desta barreira natural, a fisiologia de muitos bolores e leveduras permite sua adaptação a essas condições adversas, crescendo em substratos com concentrações de açúcares intoleráveis para as bactérias, uma vez que não são tão sensíveis às altas pressões osmóticas. ${ }^{25}$

Para estas características, Souza et al. ${ }^{2}$ relatam valores de $\mathrm{pH}$ variando entre 3,15 e 4,66, e de acidez entre 5,9 e 109,0 meq kg-1; enquanto Almeida-Anacleto ${ }^{21}$ encontrou valores variando entre 3,27 e 4,64, e 7,5 e 166,0 meq $\mathrm{kg}^{-1}$, respectivamente. Em méis da região amazônica, analisados por Almeida-Muradian et al., ${ }^{6}$ esses valores ficaram entre 3,41 e 4,06, e 20,6 e 25,3 meq $\mathrm{kg}^{-1}$. Para méis de $M$. compressipes, Oliveira et al..$^{13}$ encontraram valores médios de 3,3 para $\mathrm{pH}$ e de 91,1 meq $\mathrm{kg}^{-1}$ para acidez.

Para o índice de formol, característica também não contemplada para fiscalização do mel, a variação foi de 3,06 a $16,12 \mathrm{~mL} \mathrm{~kg}^{-1}$. Dentre os trabalhos que avaliaram esta característica em mel de meliponíneos, ${ }^{12,15,16,21}$ a variação encontrada foi de 3,00 a $21,50 \mathrm{~mL} \mathrm{~kg}^{-1}$.

Este índice é importante por representar uma medida global dos compostos aminados, permitindo avaliar o conteúdo de peptídeos, proteínas e aminoácidos. ${ }^{26}$ 


\section{Cinzas}

Os valores encontrados para cinzas variaram de 0,01 a $0,33 \%$, o que atende ao valor de referência estabelecido na legislação brasileira de, no máximo, $0,6 \%$.

Considerando os dados presentes na revisão realizada por Souza et al. ${ }^{2}$ e por Almeida-Anacleto, ${ }^{21}$ as variações para cinzas foram de 0,01 a $1,18 \%$ em méis de meliponíneos.

Este teor de cinzas expressa a riqueza do mel em minerais e se constitui em uma característica bastante utilizada para verificação de qualidade. Os minerais encontrados no mel podem ser modificados por diversos fatores, observando-se que o mel de origem floral tem menos cinzas que o mel de honeydew. ${ }^{22} \mathrm{O}$ conteúdo de cinzas relaciona-se ainda com a cor do mel, sendo verificado que quanto mais escuro é o mel mais cinzas ele contém. ${ }^{27}$

\section{Cor}

A cor das amostras de méis variou de branco a âmbar, respectivamente absorbâncias de 0,111 a 0,669. A maioria das amostras foi representada pelas cores âmbar extra claro $(48,5 \%$ das amostras) e âmbar claro $(36,4 \%)$, seguidas por âmbar $(12,1 \%)$ e branco $(3,0 \%)$. Estas cores foram determinadas em espectrofotômetro (Metrolab 1700 UvVis), a 635nm e utilizando glicerina como branco.

Na literatura são encontradas referências à ocorrência de praticamente todas as cores previstas na legislação para mel, como registrado por Iwama, ${ }^{28}$ variando de praticamente incolor a castanho escuro; âmbar claro como predominante nas amostras estudadas por Almeida ${ }^{16}$ e variações entre âmbar extra claro e âmbar, encontradas por Almeida-Anacleto. ${ }^{21}$

Esta característica do mel é a de maior influência sobre a preferência do consumidor que, na maioria das vezes, escolhe o produto apenas pela aparência.

\section{Viscosidade}

A viscosidade nas amostras variou de 10,0 a $220,0 \mathrm{mPa}$, podendo ser utilizada como indicativo da quantidade de água no produto, embora as normas de fiscalização não apresentem valores de referência. Esta característica foi determinada utilizando viscosímetro digital (Brookfield, modelo DV-I+), acoplado a banho termostático, à temperatura de $25 \pm 1{ }^{\circ} \mathrm{C}$ e $1,5 \mathrm{rpm}$.

Seemann e Neira ${ }^{29}$ afirmam que o conteúdo de água pode influenciar na viscosidade do produto. Desta forma, para méis de abelhas sem ferrão são normalmente encontrados valores baixos para a viscosidade quando comparados àqueles de A. mellifera.

\section{Condutividade elétrica}

Os valores de condutividade elétrica determinados para as amostras de mel variaram entre 255,0 e 905,7 $\mu \mathrm{S} \mathrm{cm}^{-1}$. Essa característica não apresenta valores de referência na legislação atual, ${ }^{10}$ mas é fixado pela internacional ${ }^{11} \mathrm{em}$ máximo de $800,0 \mu \mathrm{S} \mathrm{cm}^{-1}$, sendo duas amostras (14 e 22), correspondendo a 6,1\%, desclassificadas.

Os trabalhos realizados ${ }^{2,16,21}$ registram variações de 320,0 a $2700,0 \mu \mathrm{S} \mathrm{cm}{ }^{-1}$. De acordo com Bogdanov et al. ${ }^{22}$ e Stefanini, ${ }^{30}$ essa característica apresenta correlação com o conteúdo de cinzas, $\mathrm{pH}$, acidez, sais minerais, além da proteína e outras substâncias presentes no mel. É ainda relatado por $\operatorname{Campos}^{31}$ que méis de mesma origem floral apresentam condutividade elétrica muito semelhante, apesar de origens geográficas e condições climáticas diferentes.

\section{Açúcares redutores totais, açúcares redutores e sacarose}

Açúcares redutores totais, açúcares redutores e sacarose variaram nas amostras de mel entre 57,9 e 95,6\%, 50,6 e 93,1\%, e 0,2 e 9,0\%, respectivamente. Destas características somente açúcares redutores totais não está contemplado nas normas nacional e internacional. Um total de 16 amostras foi considerado reprovado pela legislação brasileira, que apresenta valores mais restritivos que a norma internacional para teores de açúcares redutores, e quatro para sacarose, respectivamente, 48,5 e $12,1 \%$ do total.

Resultados obtidos por Almeida-Anacleto ${ }^{21}$ mostraram variação de 32,4 a $66,6 \%$ (média de $55,4 \%$ ) para açúcares redutores totais, de 31,9 a $64,2 \%$ (média de 54,4\%) para açúcares redutores e de 0,1 a 2,3\% (média de $0,9 \%$ ) para sacarose. Segundo este autor, todas as amostras foram reprovadas considerando os valores de açúcares redutores apresentados e 16,1\% pelos valores de sacarose. Para Souza et al., ${ }^{2}$ valores de açúcares redutores variaram de 58,0 a 75,7\%, e sacarose de 1,1 a 4,8; enquanto para Almeida-Muradian et al., ${ }^{6}$ os valores encontrados para açúcares redutores totais variaram entre 60,2 e $61,7 \%$, para açúcares redutores de 60,2 a 61,3\% e sacarose de 0,1 a $0,2 \%$, para espécies amazônicas de abelhas sem ferrão. Ainda segundo estes autores, frutose e glicose apresentaram valores médios de 31,6 e $29,3 \%$.

Para méis de meliponíneos coletados na Venezuela, Bogdanov et $a l .{ }^{32}$ encontraram diferentes espectros de açúcares entre os gêneros destas abelhas, predominando em Melipona spp. a frutose e a glicose, e nos demais gêneros a maltose. Uma proposta de utilização destes açúcares como forma de determinar a origem entomológica dos méis de abelhas sem ferrão foi feita por Vit et al..$^{33}$

Estes açúcares monossacarídeos são os componentes em maior concentração no mel, variando de 85 a $95 \%$ da sua composição, enquanto que a sacarose, um açúcar não redutor oligossacarídeo, representa de 2 a $3 \%$ dos carboidratos presentes no mel. O aparecimento de altas concentrações deste açúcar é um indicativo de uma colheita prematura do produto, antes de uma maior ação da invertase sobre a sacarose. ${ }^{34}$ Já a sua utilização como um indicativo de adulteração do mel deve ser feita com ressalva, como verificado por Guler et al. ${ }^{35}$ em que "mel" produzido por colônias de A. mellifera alimentadas com xarope de sacarose apresentaram níveis deste açúcar tão baixos quanto os méis florais, não sendo, portanto, um bom indicativo para distinguir méis florais de méis adulterados.

\section{Hidroximetilfurfural (HMF)}

O HMF variou nas amostras de mel entre 0,0 e $60,2 \mathrm{mg} \mathrm{kg}^{-1}$, sendo que somente uma amostra, correspondendo a 3,0\% do total, excedeu o limite máximo de $60,0 \mathrm{mg} \mathrm{kg}^{-1}$ estabelecido pela legislação brasileira. ${ }^{10} \mathrm{O}$ valor determinado para esta amostra, de 60,2 mg $\mathrm{kg}^{-1}$, esteve próximo ao limite de aceitação. Considerando a norma internacional, ${ }^{11}$ e o limite de $80,0 \mathrm{mg} \mathrm{kg}^{-1}$ fixado para méis de regiões tropicais, nenhuma foi desclassificada.

Verifica-se uma variação ampla na faixa de HMF dos méis de abelhas sem ferrão, embora todos os valores obtidos até este momento ${ }^{12,15,16,18,20}$ tenham atendido ao limite máximo de $60,0 \mathrm{mg} \mathrm{kg}^{-1}$. No entanto, estudos mais recentes mostram resultados semelhantes aos encontrados nestas amostras de meliponíneos da Bahia, como o de Souza et al., ${ }^{2}$ registrando variações de 0,4 a 78,5 $\mathrm{mg} \mathrm{kg}^{-1}$ e AlmeidaAnacleto $^{21}$ de 0,8 a $71,5 \mathrm{mg} \mathrm{kg}^{-1}$.

Condições inadequadas de armazenamento e o tratamento térmico excessivo são alguns fatores que podem levar a níveis crescentes de HMF no mel de A. mellifera, sendo também afetado pela acidez, $\mathrm{pH}$, conteúdo de água e sais minerais. ${ }^{36}$ No entanto, Karabournioti e Zervalaki ${ }^{37}$ afirmam que somente a quantificação deste composto não 
é suficiente para comprovar a exposição do mel a tratamentos térmicos entre 40 e $50{ }^{\circ} \mathrm{C}$, sendo representativo somente para condições de aquecimento exagerado.

\section{Proteínas}

As proteínas variaram nas amostras entre 0,04 e 1,21\%, não existindo valores de referência para esta característica.

De maneira geral, esses teores protéicos dos méis de meliponíneos são baixos, como os registrados por Souza et al., ${ }^{2}$ apresentando variação de 0,09 a $0,90 \%$ (apresentados originalmente como $\mathrm{mg}$ nitrogênio/100 g de mel e convertidos para percentual de proteína dividindo-se por 160 , conforme apresentado por esses autores), e por Almeida-Anacleto, ${ }^{21}$ variando de 0,15 a $0,57 \%$. Valores próximos aos obtidos no presente estudo são registrados em Carvalho et al. ${ }^{17}$ variando de 0,40 a 2,84\%. Apesar do pouco conhecimento sobre as características do material protéico, a ocorrência de proteína no mel é utilizada na detecção de adulteração por produto comercial. ${ }^{38}$

\section{Atividade de água $\left(a_{w}\right)$}

As amostras de mel de meliponíneos apresentaram atividade de água variando entre 0,662 e 0,851. Esta característica, ainda pouco avaliada em méis produzidos pelas abelhas sem ferrão, tem variações registradas de 0,580 a 0,820 para méis de diversos gêneros do Estado de São Paulo, ${ }^{21}$ de 0,740 a 0,760 para espécies de Melipona amazônicas $^{6}$ e de 0,694 e 0,730 para duas espécies de Melipona da Guatemala e Venezuela. $^{3}$

A relevância desta característica está relacionada ao fato da água ser o principal componente de muitos alimentos e ter influência sobre sua estabilidade bioquímica, ${ }^{39}$ sendo atualmente uma análise utilizada para a definição de regulamento de segurança com enfoque no crescimento de microrganismos indesejáveis, definições de potencial de riscos alimentares, controle de pontos críticos, normas para alimentos em conservas e exigências de embalagem. ${ }^{8}$
Uma tabela com os valores médios obtidos para as análises físico-químicas realizadas para as espécies de Melipona, e os padrões estabelecidos pelas legislações brasileira e internacional para mel ${ }^{10,11}$ e pela proposta para mel de meliponíneos do Brasil sugerida por Villas-Bôas e Malaspina, ${ }^{40}$ é apresentada (Tabela 3).

Pela análise dos valores médios obtidos no presente trabalho, há a possibilidade do mel produzido por espécies de Melipona atender às características estabelecidas pela legislação brasileira ${ }^{10}$ e pelo Codex. ${ }^{11}$ Para isso, o principal ajuste diz respeito à elevada umidade apresentada, considerando que todas as amostras foram reprovadas por essas normas, e cinco (correspondendo a 15,2\%) reprovadas pela proposta de Villas-Bôas e Malaspina, ${ }^{40}$ com a ressalva de que esta última foi proposta justamente com o objetivo de atender às características do mel produzido por meliponíneos do Brasil.

Com relação ao número de amostras (16) que foram desclassificadas por não atenderem o nível mínimo de açúcares redutores estabelecido na legislação brasileira, ${ }^{10}$ novos limites também deverão ser ajustados de forma a melhor representar as características do mel dos meliponíneos. Deve-se ressaltar, no entanto, que este não atendimento às normas não é exclusividade do mel produzido por meliponíneos, sendo verificada presença de mel de A. mellifera também fora destes padrões.

Considerando a ausência de leitura da atividade diastásica para as amostras de mel de Melipona analisadas, e que os trabalhos publicados até o momento relatam uma baixa atividade desta enzima em mel deste gênero, atenção especial também deve ser dada aos valores estabelecidos para esta característica como também em relação ao seu uso como indicativo do frescor do mel.

\section{CONSIDERAÇÕES FINAIS}

As amostras de mel de espécies de Melipona colhidas no Estado da Bahia apresentaram valores médios para as características físicoquímicas, atendendo à maioria dos pré-requisitos estabelecidos pelas regulamentações brasileira e internacional para fiscalização do mel,

Tabela 3. Características físico-químicas médias determinadas para mel de Melipona (Apidae: Meliponini) do Estado da Bahia, região nordeste do Brasil, e comparação com os valores de referência estabelecidos nas regulamentações vigentes para fiscalização de mel ${ }^{10,11}$ e a proposta para mel de meliponíneos do Brasil ${ }^{40}$

\begin{tabular}{|c|c|c|c|c|c|c|c|c|c|c|c|c|c|c|c|}
\hline \multirow{2}{*}{ Espécies } & \multirow{2}{*}{$\begin{array}{l}\text { Número } \\
\text { de } \\
\text { amostras }\end{array}$} & \multicolumn{14}{|c|}{ Características físico-químicas* } \\
\hline & & Umi & $\mathrm{pH}$ & Aci & IF & Cin & Cor & Vis & Cond & AT & $\mathrm{AR}$ & $\mathrm{Sac}$ & HMF & Pro & $a_{w}$ \\
\hline Melipona spp. & 33 & 31,9 & 4,08 & 33,9 & 6,80 & 0,14 & 0,277 & 81,5 & 542,0 & 71,1 & 68,4 & 2,5 & 10,3 & 0,27 & 0,742 \\
\hline M. asilvai & 07 & 37,5 & 3,55 & 54,2 & 7,34 & 0,09 & 0,245 & 25,7 & 546,5 & 65,3 & 61,7 & 3,3 & 14,7 & 0,33 & 0,792 \\
\hline M. mandacaia & 02 & 31,4 & 3,71 & 37,7 & 8,53 & 0,09 & 0,249 & 100,0 & 283,7 & 78,6 & 75,5 & 2,9 & 30,9 & 0,17 & 0,749 \\
\hline $\begin{array}{l}\text { M. quadrifasciata } \\
\text { anthidioides }\end{array}$ & 09 & 32,1 & 3,99 & 40,6 & 5,14 & 0,10 & 0,413 & 47,8 & 548,8 & 71,5 & 68,3 & 3,1 & 16,0 & 0,29 & 0,761 \\
\hline M. scutellaris & 15 & 29,1 & 4,43 & 19,9 & 7,31 & 0,19 & 0,213 & 125,3 & 570,2 & 72,6 & 70,7 & 1,8 & 2,0 & 0,25 & 0,707 \\
\hline \multicolumn{16}{|c|}{ Valores de referência } \\
\hline Brasil $^{10}$ & & $\begin{array}{l}\text { máx. } \\
20,0\end{array}$ & - & $\begin{array}{l}\text { máx. } \\
50,0\end{array}$ & - & $\begin{array}{l}\text { máx. } \\
0,60\end{array}$ & - & - & - & - & $\begin{array}{l}\text { mín. } \\
65,0\end{array}$ & $\begin{array}{c}\text { máx. } \\
6,0\end{array}$ & $\begin{array}{l}\text { máx. } \\
60,0\end{array}$ & - & - \\
\hline Codex ${ }^{11}$ & & $\begin{array}{l}\text { máx. } \\
20,0\end{array}$ & - & $\begin{array}{l}\text { máx. } \\
50,0\end{array}$ & - & - & - & - & $\begin{array}{l}\text { máx. } \\
800,0\end{array}$ & - & $\begin{array}{l}\text { mín. } \\
60,0\end{array}$ & $\begin{array}{c}\text { máx. } \\
5,0\end{array}$ & $\begin{array}{l}\text { máx. } \\
80,0^{* * *}\end{array}$ & - & - \\
\hline Villas-Bôas e Mala & & $\begin{array}{l}\text { máx. } \\
35,0\end{array}$ & & $\begin{array}{l}\text { máx. } \\
85,0\end{array}$ & & $\begin{array}{c}\text { máx. } \\
0,6\end{array}$ & - & - & - & - & $\begin{array}{l}\text { mín. } \\
50,0\end{array}$ & $\begin{array}{c}\text { máx. } \\
6,0\end{array}$ & $\begin{array}{c}\text { máx. } \\
40,0\end{array}$ & - & - \\
\hline
\end{tabular}

*Valores médios. **Para méis provenientes de regiões tropicais. Umi: Umidade (\%); Aci: Acidez livre (meq kg-1 $)$; IF: Índice de formol (mL $\mathrm{kg}^{-1}$ ); Cin: cinzas (\%); Vis: Viscosidade (mPa s); Cond: Condutividade elétrica ( $\left.\mu \mathrm{cm}^{-1}\right)$; AT: Açúcares redutores totais $(\%)$; AR: Açúcares redutores (\%); Sac: Sacarose (\%); HMF: Hidroximetilfurfural $\left(\mathrm{mg} \mathrm{kg}^{-1}\right)$; Pro: Proteína $(\%)$; $a_{w}$ : atividade de água. 
à exceção da umidade que foi elevada para todas as amostras. Esta elevada umidade pode vir a se constituir em um fator de redução na vida de prateleira do produto, devido à ocorrência de processos fermentativos.

Apesar de apresentar valores médios atendendo às regulamentações para mel, os limites para açúcares redutores devem ser melhor ajustados para mel de meliponíneos considerando que, individualmente, quase metade das amostras esteve fora dos limites estabelecidos para esta característica.

A utilização da atividade diastásica não é o melhor critério para a definição do frescor do mel, considerando a ausência de leitura da atividade desta enzima em mel de Melipona.

\section{AGRADECIMENTOS}

Ao CNPq pela concessão de bolsa de doutorado ao primeiro autor, e de Produtividade em Pesquisa aos segundo e quarto autores; e à FAPESP por auxílio financeiro concedido.

\section{REFERÊNCIAS}

1. Fallico, B.; Zappalà, M.; Arena, E.; Verzera, A.; Food Chem. 2004, 85, 305.

2. Souza, B. A.; Roubik, D. W.; Barth, O. M.; Heard, T. A.; Enríquez, E.; Carvalho, C. A. L.; Villas-Bôas, J. K.; Marchini, L. C.; Locatelli, J. C.; Persano-Oddo, L.; Almeida-Muradian, L. B.; Bogdanov, S; Vit, P.; Interciencia 2006, 31, 867.

3. Vit, P.; Enriquez, E.; Barth, M. O.; Matsuda, A. H.; Almeida-Muradian, L. B.; RFM 2006a, 15, 89.

4. Vit, P.; Rodríguez-Malaver, A.; Almeida, D.; Souza, B. A.; Marchini, L. C.; Díaz, C. F.; Tricio, A. E.; Villas-Bôas, J. K.; Heard, T. A.; Magistra 2006b, 18, 270.

5. Almeida-Muradian, L. B.; Barion, F.; Proceedings $40^{\text {th }}$ Apimondia International Apicultural Congress, Melbourne, Austrália, 2007.

6. Almeida-Muradian, L. B.; Matsuda, A. H.; Bastos, D. H. M.; Quim. Nova 2007, 30, 707.

7. http://www.atago.net, acessada em Novembro 2007; Codex Alimentarius Commission; Codex Alimentarius 1990, 3, 1; Moraes, R. M.; Teixeira, E. W.; Análise do mel (Manual técnico), Pindamonhangaba, 1998; Moraes, R. M.; Análise de mel, Pindamonhangaba, 1994; Pregnolato, W.; Normas analíticas do Instituto Adolfo Lutz, São Paulo, 1985; Bianchi, E. M.; Control de calidad de la miel, Santiago del Estero, 1986; American Society for Testing and Materials; American Society for Testing and Materials, Barr Harbor, [200-]; Boletin Oficial Español; Boletin Oficial Español, Madrid, 1986; Marchini, L. C.; Sodré, G. S; Moreti, A. C. C. C.; Mel brasileiro: composição e normas, Ribeirão Preto, 2004; Association of Official Analytical Chemists; Official methods of analysis, Arlington, 1992; Silva, D. J.; Queiroz, A. C. Em Análise de alimentos: métodos químicos e biológicos; Silva, D. J.; Queiroz, A. C., eds.; Viçosa, 2002, p. 57; Decagon; Aqualab - water active meter: operator's manual, Washington, 2005.

8. Scott, V. N.; Clavero, R. S.; Troller, J. A. Em Compendium of methods for the microbiological examination of foods; Downes, F. P.; Ito, K., eds..; Washington, 2001, p. 649.

9. Bogdanov, S.; Martin, P.; Lullmann, C.; Apidologie 1997, 2, 1.

10. http://www.agricultura.gov.br, acessada em Março 2008.
11. http://www.codexalimentarius.net, acessada em Dezembro 2007.

12. Souza, B. A.; Carvalho, C. A. L.; Sodré, G. S.; Marchini, L. C.; Cienc. Rural 2004a, 34, 1623.

13. Oliveira, E. G.; Monteiro Neto, V.; Silveira, L. M. S.; Nascimento, A. R.; Nahuz, M. S. R.; Meneses, S. L.; Vasconcelos, A. F. F.; Costa, M. C. P.; Borges, A. C. S.; Bogéa, A. L. G.; Azevedo, C. C.; Ferreira, C. F. C.; Lima, J. C.; Hig. Aliment. 2006, 20, 74.

14. Bijlsma, L.; Bruijn, L. L. M.; Martens, E. P.; Sommeijer, M. J.; Apidologie 2006, 37, 480.

15. Alves, R. M. O.; Carvalho, C. A. L.; Souza, B. A.; Sodré, G. S.; Marchini, L. C.; Ciênc. Tecnol. Aliment. 2005, 25, 644.

16. Almeida, D.; Dissertação de Mestrado, Universidade de São Paulo, Brasil, 2002.

17. Carvalho, C. A. L.; Souza, B. A.; Sodré, G. S.; Marchini, L. C.; Alves, R. M. O.; Mel de abelhas sem ferrão: contribuição para a caracterização físico-química, Cruz das Almas: Bahia, 2005.

18. Evangelista-Rodrigues, A.; Silva, E. M.; Beserra, E. M.; Rodrígues, M. L.; Cienc. Rural 2005, 35, 1166.

19. Souza, R. C. S.; Yuyama, L. K. O.; Aguiar, J. P. L.; Oliveira, F. P. M.; Acta Amazon. 2004b, 34, 333.

20. Rodrigues, A. C. L.; Marchini, L. C.; Carvalho, C. A. L.; Rev. Agric. 1998, 73, 255.

21. Almeida-Anacleto, D.; Tese de Doutorado, Universidade de São Paulo, Brasil, 2007.

22. Bogdanov, S.; Lüllmann, C.; Martin, P.; Ohe, W. von der; Russmann, H.; Vorwohl, G.; Persano-Oddo, L.; Sabatini, A. G.; Marcazzan, G. L.; Piro, R.; Flamini, C.; Morlot, M.; Lhéritier, J.; Borneck, R.; Marioleas, P.; Tsigouri, A.; Kerkvliet, J.; Ortiz, A.; Ivanov, T.; D’Arcy, B.; Mossel, B.; Vit, P.; Bee World 1999, 80, 61.

23. Vit, P.; Pulcini, P.; J. Apic. Rres. 1996, 35, 57.

24. White Júnior, J. W.; Bee World 1994, 75, 104.

25. Lacaz-Ruiz, R.; Manual prático de microbiologia básica, São Paulo, 2000.

26. Temiz, A. I.; CAB Abstracts on CD-ROM, 1984.

27. Ortiz, V. A.; Apicultural Abstracts, 1989.

28. Iwama, S.; Dissertação de Mestrado, Universidade de São Paulo, Brasil, 1977.

29. Seemann, P.; Neira, M.; Tecnología de la produccíon apícola, Valdivia: Chile, 1988.

30. Stefanini, R.; Apiacta 1984, 19, 109.

31. Campos, G.; Tese de Doutorado, Universidade Federal de Minas Gerais, Brasil, 1998.

32. Bogdanov, S.; Vit, P.; Kilchenmann, V.; Apidologie 1996, 26, 445.

33. Vit, P.; Fernandez-Maeso, M. C.; Ortiz-Valbuena, A.; J. Appl. Eentomol. 1998, 122, 58.

34. Crane, E.; O livro do mel, Nobel: São Paulo, 1985

35. Guler, A.; Bakan, A.; Nisbet, C.; Yavuz, O.; Food Chem. 2007, 105 , 119.

36. White Júnior, J. W. Em The hive and honeybee; Graham, J., ed.; Dadant \& Sons: Hamilton, 1976.

37. Karabournioti, S.; Zervalaki, P.; Apiacta 2001, 36, 177.

38. Crane, E.; Honey: a comprehensive survey, Heinemann: London, 1975.

39. Gleiter, R. A.; Horn, H.; Isengard, H. D.; Food Chem. 2006, 96, 441.

40. Villas-Bôas, J. K.; Malaspina, O.; Mensagem Doce 2005, 82, 6. 